\title{
Comparison between accelerometer and laser vibrometer to measure traffic excited vibrations on bridges
}

\author{
G. Rossi ${ }^{\mathrm{a}}$, R. Marsili ${ }^{\mathrm{a}}$, V. Gusella ${ }^{\mathrm{b}}$ and M. Gioffrè ${ }^{\mathrm{b}}$ \\ ${ }^{a}$ Dip. di Ingegneria Industriale - Università degli Studi di Perugia, Via G. Duranti $n^{\circ} 1 / A-4-06125$ Perugia, Italy \\ Tel.: +3975 5853744; Fax: +3975 5853703; E-mail: misure@ unipg.it \\ ${ }^{\mathrm{b}}$ Dip. di Ingegneria Civile e Ambientale - Università degli Studi di Perugia, Italy
}

\begin{abstract}
The use of accelerometer based measurement techniques for evaluating bridge forced vibrations or to perform bridge modal analysis is well established. It is well known to all researchers who have experience in vibration measurements that values of acceleration amplitude can be very low at low frequencies and that a limitation to the use of accelerometer can be due to the threshold parameter of this kind of transducer. Under this conditions the measurement of displacement seems more appropriate. On the other hand laser vibrometer systems detect relative displacements as opposed to the absolute measures of accelerometers. Vibrations have been measured simultaneously by a typical accelerometer for civil structures and by a laser vibrometer equipped with a fringe counter board in terms of velocity and displacements. The accelerations calculated from the laser vibrometer signals and the one directly measured by the accelerometer has been compared.
\end{abstract}

Keywords: Laser Doppler vibrometer, bridge vibrations, bridge health monitoring, bridge dynamic testing

\section{Introduction}

The study of the dynamic response of bridges under normal traffic conditions using experimental results is very attractive for several reasons. When dealing with new constructions it is possible to verify that the structure have the desired behavior and to validate hypothesis and models assumed in the design stage. Even more significant is the analysis of experimental tests on pre-existing bridges because of the increased magnitude of the loads from those assumed in the design stage. This is due both to the increased traffic intensity and frequency and to the new seismic classification of the territory. Furthermore, if the natural impairment under the working conditions of the structure is considered, it is clear that a reliable estimate of the safety under working and limit loads is a very complex task. The response analysis of bridges under traffic loads allow the identification of analytical and/or numerical models, which can be used for estimating the behavior under peak loads.
Important information on the load model and associated response can be obtained by studying the effect of mechanical excitations, pulse forces, and traffic on the bridge fluctuations. This is of interest to perform a damage analysis due to load cycles. This kind of analysis require an high degree of reliability and the repeated execution of the tests in different times. It follows that the experimental techniques and procedures have to be carefully chosen in order to be not intrusive, easily repeatable, and able to provide a reliable characterizations of the bridge dynamic behavior. Within this context, the laser vibrometry appears to be very attractive for applications because it allows measurements at distance and with no contact. Current vibrometers allow measurements up to $100 \mathrm{~m}$ placing appropriate paint or reflecting stripes on the target. The first results of the experimental work on a reinforced concrete bridge are presented in this paper focusing on the comparisons between vibrometer and accelerometer measurements showing the higher accuracy of the 
laser measurements. Furthermore the vibrometer provides directly the bridge inflection, which represent the most interesting parameter for the structural analysis.

\section{A theoretical reference model}

The analytical solution of the dynamic response of a bridge under moving loads is a very complex task because it is affected by several factors and many theoretical models have been proposed in literature9. As an example, the specific case of a simply supported beam is reported (see Fig. 1). The length of the beam is $l$ and a harmonic force $P(t)$ is assumed to move at constant velocity $V_{0}$. The analytical solution of this problem has been first proposed by S.P. Timoshenko and then developed in many works by L. Fryba. Figure 2 (from [9]) shows the solution when the force frequency is lower, equal and higher than the beam first resonance.

The theoretical results illustrated here show that the maximum static peak value can be amplified by the superimposed harmonic components. This amplification is usually described by the dynamic load amplification factor, which is defined as the ratio between the maximum dynamic load and the maximum static load. Experimental tests with strain transducers on five steel girder highway bridges demonstrated that the displacement waveforms are similar to those of Fig. 2 [10]. Furthermore is has been seen that the dynamic loads can represent an important component of the bridge loads and depend on the vehicle type, weight, configuration, bridge span length, road roughness and transverse position of vehicle on the bridge. More sophisticated models need therefore to describe the vehicle dynamic characteristics [9]. On the other hand some of the factors like the roughness and the imperfections of the road make the application of these analytical models rather complex.

To overcome these difficulties Finite Element based (FEM) numerical models have been proposed, which try to describe the actual bridge structural scheme but they still show a lack of accuracy on some of the issues outlined above.

These analytical and/or numerical models can be safely used in the bridge analysis for peak loads if they are validated by experimental results. This is true especially when dealing with pre-existing structures, which geometrical, mechanical, and support characteristics are affected by high variability.

\section{Typical bridge vibrations}

The need of dynamic tests on highway bridges was already felt in the years between 1892 and 1913 in Switzerland but the most part of the experimental work on bridges was performed after the second World War. Theoretical and experimental developments have been extensively reported in literature.

The methods to excite bridge vibrations fall into two general categories: 1) measured input test (weight drop on rubber buffer, explosive charge, impact hammer, explosive cut tensioned cable, electrodynamic and hydraulic exciters, etc); 2) ambient tests (normal traffic, wind, or test vehicles on the bridge) [1].

Using a simple but clearly not exhaustive approach, bridges can be divided into two categories: large and small bridges. For example, large bridges are the cable-stayed bridges, while highway reinforced concrete bridges with typical span between 20 and $30 \mathrm{~m}$ are considered small. Wind and traffic are the only practical excitation techniques for large bridges.

FEM can be used to estimate mode shapes and structure damping, which can be used to find the optimal location to place the accelerometers [2] in the experimental tests. The optimal range in the number of accelerometers have been found to be $6-18$ for bridges with 250-330 $\mathrm{m}$ span [3]. The most interesting modes are usually the first three, corresponding to bending (on the horizontal and vertical plane) and torsion. Large bridges have frequencies in the range of $0.2-2 \mathrm{~Hz}$ associated to these modes, which are usually very close one another and difficult to distinguish by the common FFT processing technique. The use of narrow band filters (bandwidth $0.1-0.04 \mathrm{~Hz}$ ) helps to overcome this problem and to estimate the damping in the time domain [2]. Characteristic critical damping values in large bridges are in the range $0.4-2.4 \%$, which suggest the need of long records for their estimations (usually 10 minutes [4]).

The interesting modes in small bridges have frequencies in the range of 3-30 Hz [5-7], which can be determined by the common spectral estimation techniques. The amplitudes recorded during dynamic tests are in the order of millimeters (centimeters with full load) with typical values of 2-3 mm produced by heavy trucks on a $25 \mathrm{~m}$ span highway bridge [5].

In the last few years bridge vibration measurement have been proposed to perform health monitoring of the structure [7] because bridges are still used after decades despite ageing and the associated fatigue damage accumulation. The possibility to monitor their health state 


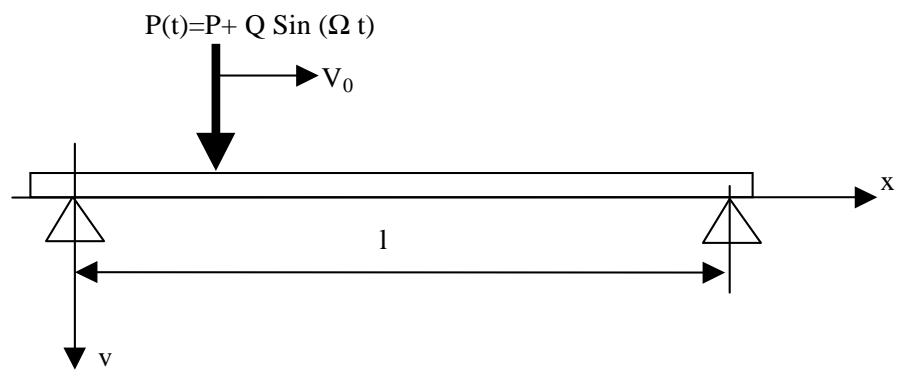

Fig. 1. Theoretical reference model.

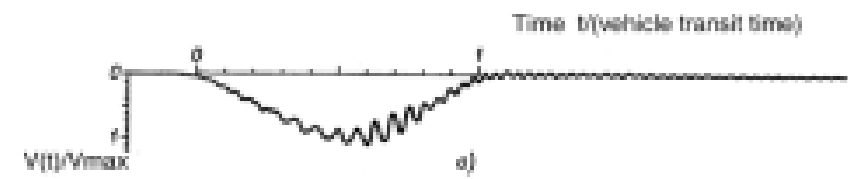

Time t/vehiche Irafsin tims:
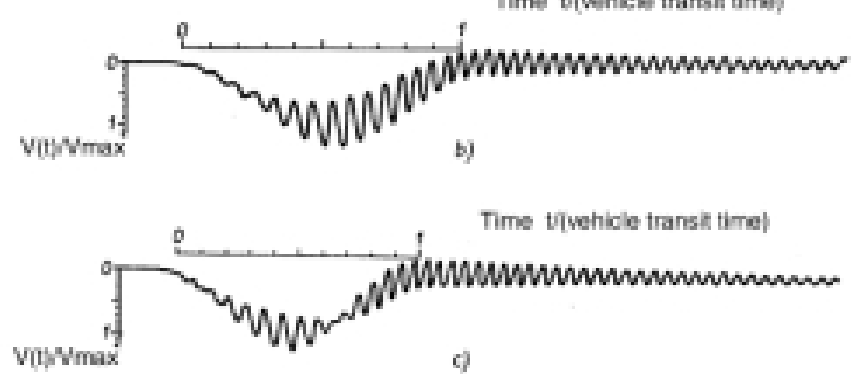

Fig. 2. Theoretical solution for beam midpoint displacement when a sine plus constant load travel on it at constant speed and frequency of exiting force is lower that first beam resonance (upper), equal to beam resonance (medium), higher than beam resonance (lower).

is therefore becoming increasingly important. Damage identification based on changes in the vibration characteristics is one of the few methods that are able to monitor the structure's global changes. The possibility to separate the effects that fatigue damage and disturbing inputs (humidity, wind but mainly temperature) have on the dynamic parameters values has been recently reported [7]. The results reported in [8] are very promising and it is this authors' opinion that the development of new and more accurate measurement techniques is the natural path to follow to improve bridge reliability.

\section{Metrological comparison: Vibrometer vs accelerometer}

The larger part of the experimental studies on bridge dynamics has been performed using accelerometers. Only recently [11] the use of a laser Doppler vibrometer has been proposed to measure tension (from resonance frequency) on cables of Weirten-Steubenville cable-
Table 1

\begin{tabular}{ll}
\hline Frequency range $(10 \%) \mathrm{Hz}$ & $0.1-1000$ \\
Max acceleration $\left(\mathrm{m} / \mathrm{s}^{2}\right)$ & $10-20$ \\
linearity \% FS & $1 \%$ \\
Sensitivity $(\mathrm{V} / \mathrm{g})$ & $1-3$ \\
Resolution - Threshold $\left(\mathrm{m} / \mathrm{s}^{2}\right)$ & $0.0001-0.0004$ \\
\hline
\end{tabular}

stayed bridges that crosses the Ohio river between Ohio and West Virginia. Typical characteristics of nowadays available piezoelectric seismic accelerometers are summarized in the Table 1.

From the frequency range specification these accelerometers seems to cover all the needs both for large and little bridge previously described. But the lowest dynamical bridge displacements that can be measured with these transducers, taking the best threshold of $0.0001 \mathrm{~m} / \mathrm{s}^{2}$ of are of about $0.25 \mathrm{~mm}$ at $0.1 \mathrm{~Hz}$. With the less sensitive transducers available this become about $1 \mathrm{~mm}$, which is not tolerable for some applications. Lowest displacements can be measured at higher frequencies, for example at $1 \mathrm{~Hz}$, using the best transducers available, it become about $2.5 \mu \mathrm{m}$. Resolution for 

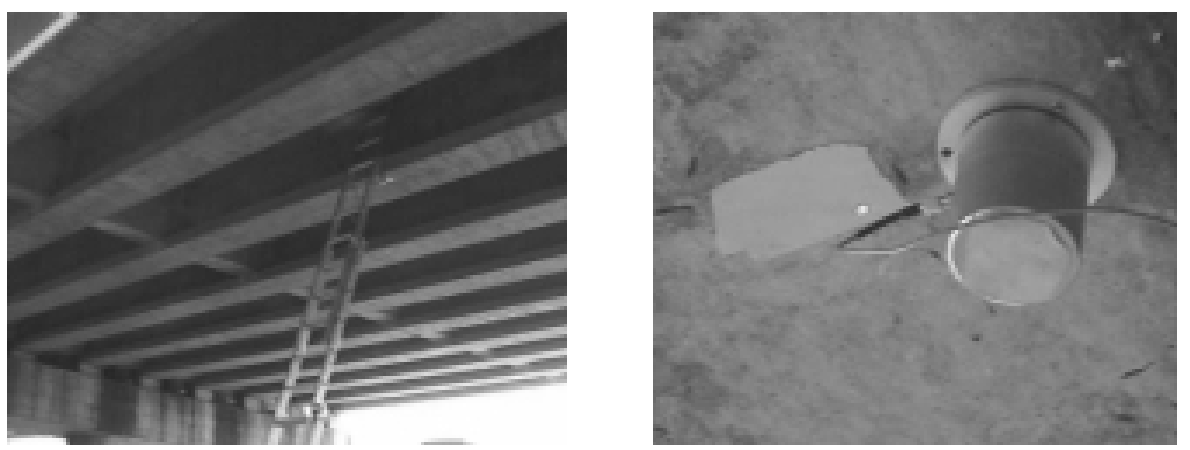

Fig. 3. The lower deck of the bridge and the measurement point.
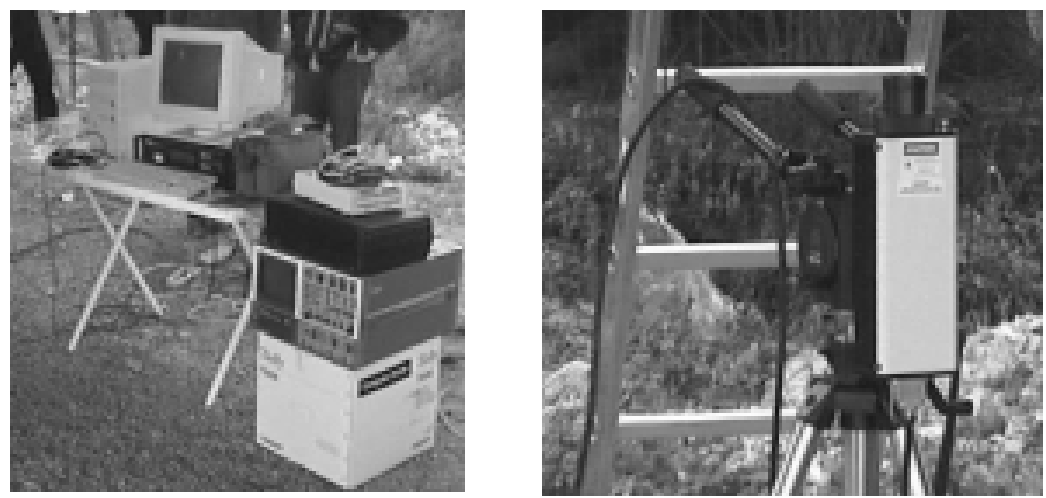

Fig. 4. Laser Doppler vibrometer measurement head and chain.

common servo-accelerometers commercially available is of the same order of magnitude (about $0.0001 \mathrm{~m} / \mathrm{s}^{2}$ ) so the above limitations do not change significantly.

Resolution of the laser Doppler vibrometer used for the present work is, in the best condition of surface reflectivity, $0.25 \mathrm{~nm}$, constant in the whole frequency range from $0 \mathrm{~Hz}$ to $250 \mathrm{kHz}$, because this instrument directly measures displacements. The advantages in metrological terms are therefore obvious: at frequencies below $1 \mathrm{~Hz}$ an uncertainty component due to threshold and resolution better of at least 1000 times can be obtained.

The difference to takes into account is that accelerometers measure absolute structure movements and laser vibrometer measure differential displacements between its optical head and the bridge, so vibrations of vibrometer head support must be considered with care in choosing its location and in the subsequent data analysis. A possible practical solution is to consider the output vibrometer signal with no dynamic loads applied to the structure as the noise background. This output is due to support vibration and other noise sources.

\section{Test performed}

The tested structure is a typical girder highway bridge with $20 \mathrm{~m}$ span. The structure is composed of 10 pre-stressed concrete beams supporting the road. The measurements illustrated here have been recorded on the mid span of the third beam that is the center beam of the most right road lane. A PCB 393c seismic piezoelectric accelerometer with $1 \mathrm{~V} / \mathrm{g}$ of sensitivity, $0.000036 \mathrm{~g}$ rms of threshold, $2.5 \mathrm{~g}$ of range, $0.01-$ $1200 \mathrm{~Hz}$ frequency range have been used. The beams and the measurement point are illustrated in Fig. 3.

Velocity and displacements have been simultaneously recorded by the Polytec OFV 3001 - OFV 303 laser Doppler vibrometer with fringe counter board OFV-600 on a PC. Figure 3 shows the laser measurement points (on and beside the accelerometer) and Fig. 4 the laser measurement head and chain.

The vibrometer velocity output signal and the accelerometer output signal have been simultaneously sampled by a Ono-Sokki CF 940 spectrum analyser. The vibrometer velocity output has been used to synchronise the displacements measured by the fringe 

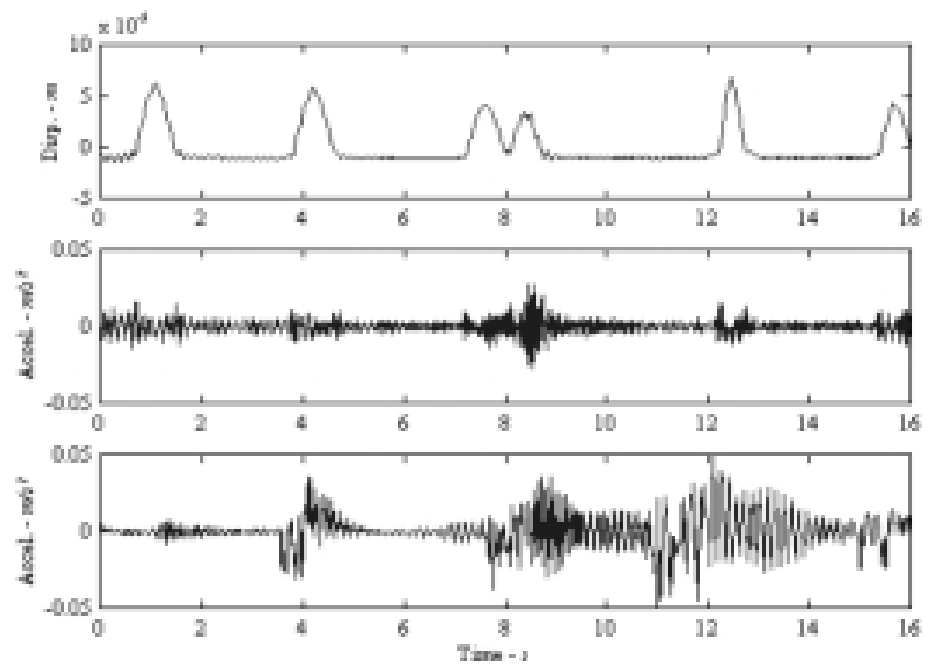

Fig. 5. Comparison between direct measurement of displacement (upper plot) by laser vibrometer, its second derivative (middle plot) and accelerometer signal (lower plot).
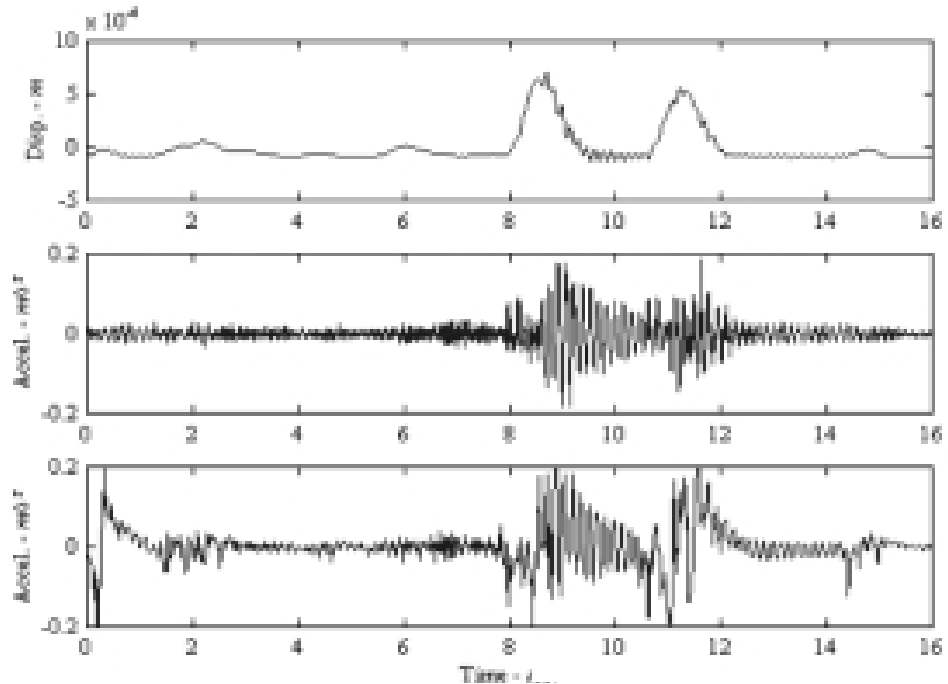

Fig. 6. Comparison between direct measurement of displacement (upper plot) by laser vibrometer, its second derivative (middle plot) and accelerometer signal (lower plot).

counter board inside the PC. The vehicle flow over the bridge during the tests have been recorded by a TV camera. The camera counter has been synchronised with the PC and Spectrum analyser clocks. The duration of the measured displacements was 16 and 128 s using sampling frequencies of 256 and $128 \mathrm{~Hz}$ respectively.

\section{Result analysis}

The measurements constitute of two sets of data. The first one considers 16 seconds time histories. The ob- tained results allow a comparison between the vibrometer and the accelerometer measurements. Figures 5 and 6 show an example of the recorded data. The first plot is the displacement obtained by the vibrometer. The second plot is the acceleration obtained deriving the displacement signal. Finally in the third plot is reported the accelerometer measurement.

Figures 7 and 8 show two typical record of the second set of measurements concerning $126 \mathrm{~s}$ time histories. These records assume noticeable importance because they provide the vertical displacement time series as a 


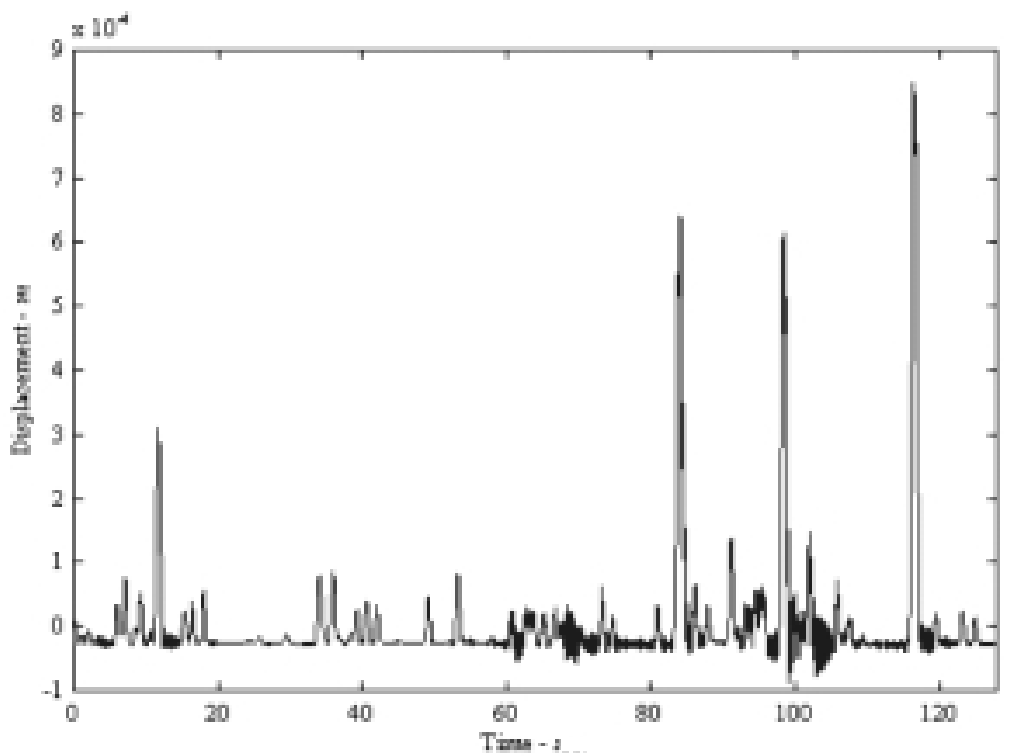

Fig. 7. Typical long time displacement record measured by the vibrometer.

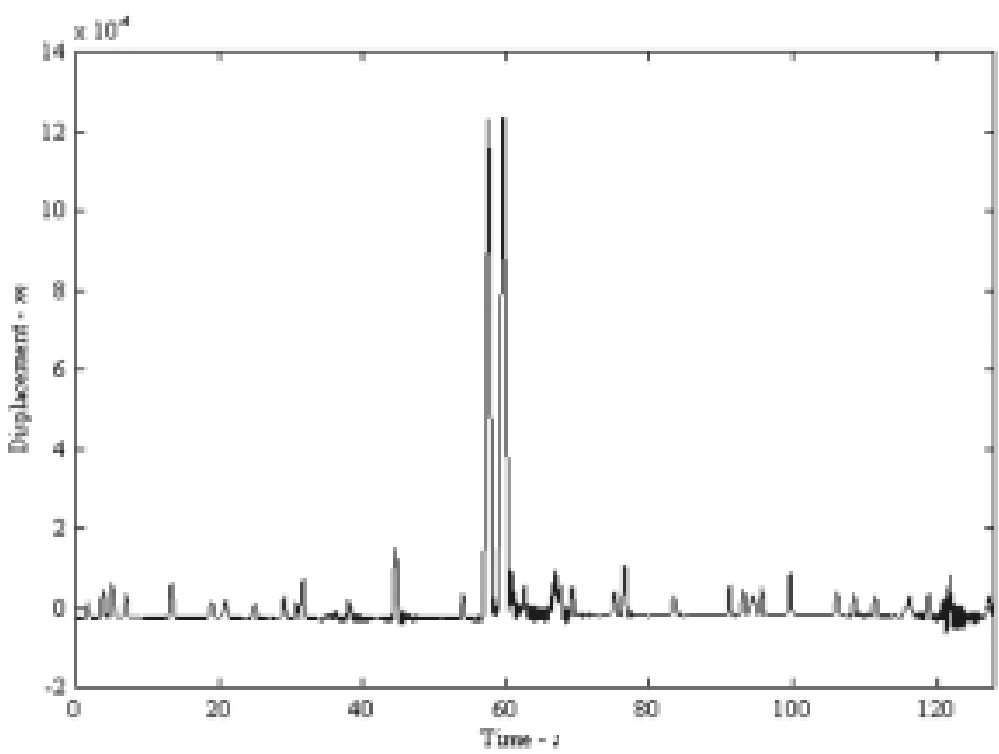

Fig. 8. Typical long time displacement record measured by the vibrometer.

function of the traffic conditions. A different behavior is evident when light or heavy vehicles are traveling on the bridge. In the first case the displacement is about $0.3 \mathrm{~mm}$ while it rise up to about $2 \mathrm{~mm}$ in the second. This information can be effectively used both for the traffic stochastic modeling and for the estimate of fatigue damage under load cycles.

A few comments can be outlined. The displacement signal provide the possibility to count the travelling cars and trucks over the bridge while this is not possible with the acceleration signal, directly or from its integrated signal because of the poor signal to noise ratio. The accelerometer do not allow accurate measurements of displacement due to light car travelling over the bridge. Even if the threshold specification and resolution can be considered sufficient for this application the effect of electrical noise, cable motion and consequent tribological electrical effects, drift on electrical 

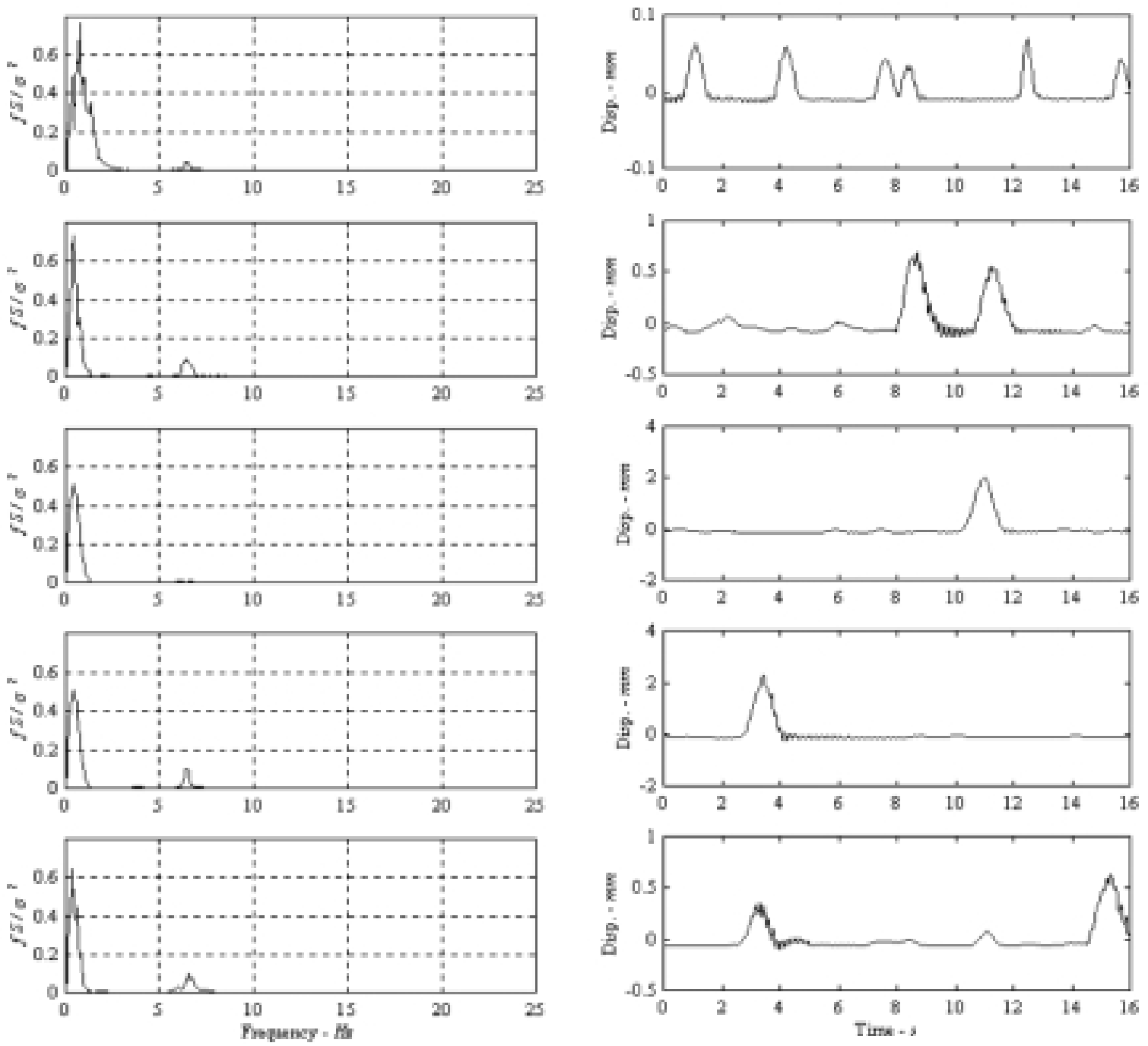

Fig. 9. Typical power spectrum of the 16 seconds records of vibrometer displacement output and corresponding time signals. The peak at $\sim 6.5 \mathrm{~Hz}$ corresponds to the natural frequency of the bridge excited by the traffic time history.

circuits to process accelerometer signal output (of few millivolt) strongly affect the result and overestimate of about $40 \%$ peak values. These effects are illustrated in Figs 5 and 6.

The typical recorded displacement signals are similar to the theoretical model, i.e. composed by a superposition of a peak and a damped harmonic function. The harmonic component correspond to the natural frequency of the bridge (vertical bending mode) and not that of the exciting force transmitted by the wheels.

This is evident from the observation of the power spectral densities of Fig. 9 which show a sharp spike at about $6 \mathrm{~Hz}$ in all the signals. The spectra also show other frequencies which differs from a vehicle passage to another.

The differences between the accelerometer and the laser vibrometer in estimating the resonance amplitude when the same vehicle is traveling are less evident and are in the order of $20 \%$.

\section{Conclusions}

The possibility to obtain highly accurate bridge displacement vibrations using a laser Doppler vibrometer has been demonstrated. The better quality of these measurements was outlined by comparison with the accelerometer results. The main advantages are due to 
the relative accuracy, which is independent by the vibration frequency, to the immunity from electrical nise and absence of cable with low electrical signal. A second advantage is the possibility to record time histories at many locations in a rather simple way since it is not necessary to install a transducer on the measurement point. All of this can open up new possibilities for analysing quickly and easily bridge structures and use the results for a better identification of the analytical and numerical models for design and structure maintenance. The improved models can also be a more accurate source of information to perform health monitoring, i.e. to check and prevent damage. On going research of the authors deals with the comparison between the experimental data and the one obtained by using the bridge numerical and analytical models.

\section{References}

[1] C.R. Farrar, T.A. Duffey, P.J. Cornwell and S.W. Doebling, Excitation methods for bridge structures, Proceedings of the XVI International Modal Analysis Conference, Santa Barbara, California, 1998.

[2] A. Cunha, E. Caetano, R. Calcada and R. Delgato, Modal identification and correlation with finite element parameters of Vasco de Gama bridge, Proceedings of the XVII International Modal Analysis Conference, Kissimmee, Florida, 1999.
[3] J. Rodrigues and A.C. Costa, Modal analysis from ambient vibration survay of bridges: Lnec experience, Proceedings of the XVII International Modal Analysis Conference, Kissimmee, Florida, 1999.

[4] S.W. Smith and M. Johnson, Field test to determine frequencies of bridge stay cables, Proceedings of the XVII International Modal Analysis Conference, Kissimmee, Florida, 1999.

[5] P.O. Almeida, P.B. Fusco and E. Penner, Diagnosis of reinforced concrete bridges by means of dynamic testing, Proceedings of the XVII International Modal Analysis Conference, Kissimmee, Florida, 1999.

[6] M.S. Lenett, D.L. Brown, V.J. Hunt and A.J. Helmicki, Performing bridge condition assesment with spliced multireference impact data, Proceedings of the XVIII International Modal Analysis Conference, San Antonio, Texas, 2000.

[7] B. Peeters and G. De Roeck, One year monitoring of the Z24 bridge: Environmental influences versus damage events, $\mathrm{Pro}$ ceedings of the XVIII International Modal Analysis Conference, San Antonio, Texas, 2000.

[8] R.G. Rohrmann, M. Baessler, S. Said, W. Schmid and W. Ruecker, Structural causes of temperature affected modal data of civil structures obtained by long time monitoring, Proceedings of the XVIII International Modal Analysis Conference, San Antonio, Texas, 2000.

[9] L. Friba, Vibration of solids and structures under moving loads, Noordhoff International Publishing, Groningen.

[10] A.S. Nowak, S. Kim and M.M. Szerszen, Dynamic loads for steel girder bridges, Proceedings of the XVII International Modal Analysis Conference, Kissimmee, Florida, 1999.

[11] J. Naiman, Laser vibrometers simplifying bridge condition evaluation, OE Reports 173, May 1998, SPIE Web, www.spie.org/web/oer/may/may98/laservib.html. 

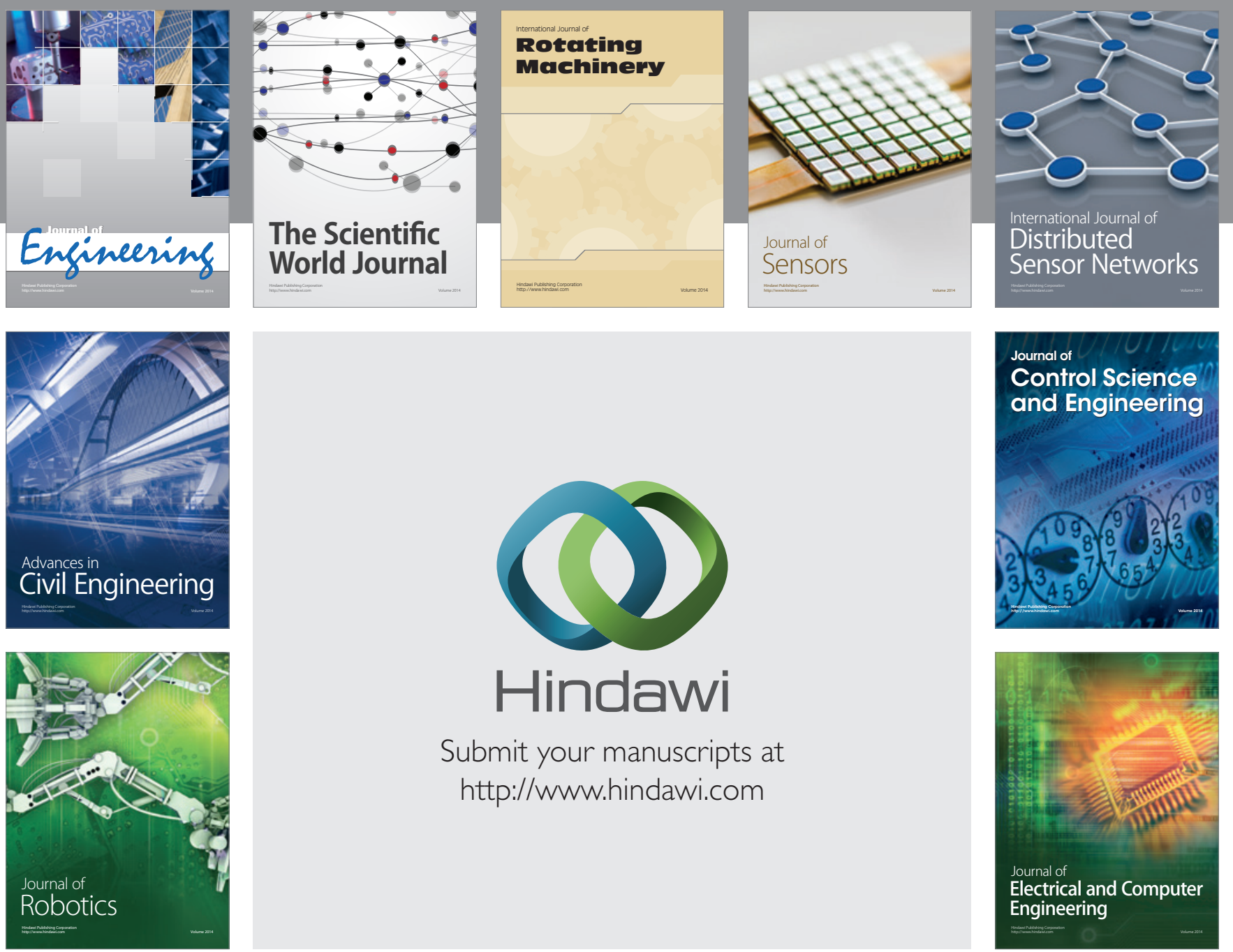

Submit your manuscripts at

http://www.hindawi.com
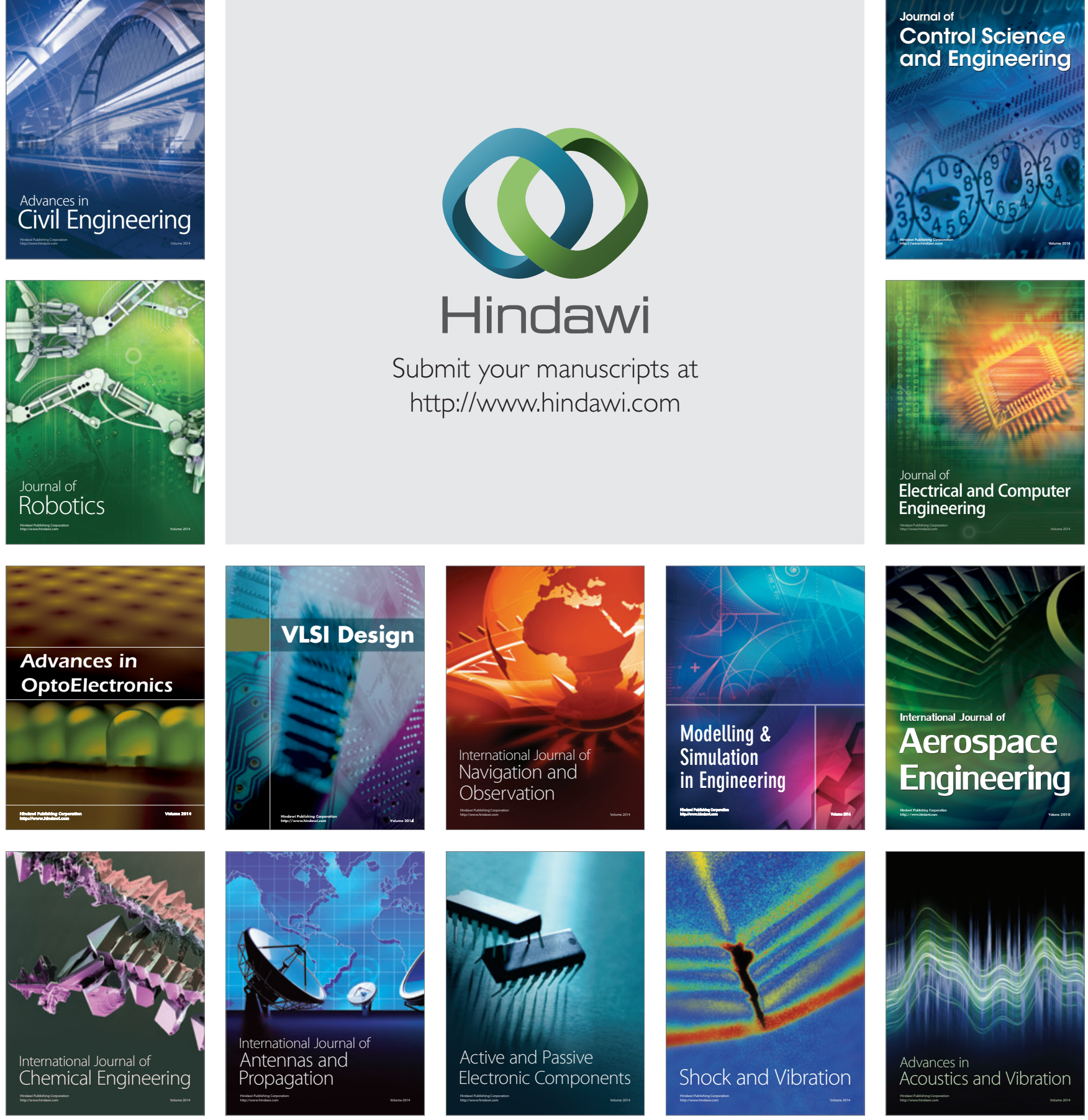